\title{
Zusammenfassung und Ausblick
}

Der Dienstleistungssektor ist der größte und am schnellsten wachsende Wirtschaftszweig in Deutschland (BMWI, 2020). Typische Dienstleistungen sind dabei unter anderem Lieferdienste, Reinigungsdienste oder Transportdienstleistungen. Bei der Betrachtung einer Dienstleistung ist es wichtig den ,Service Encounter' zu beachten. Dieser Dreiklang bei der Dienstleistungsbegegnung beschreibt die Interaktion zwischen Kunden (Nachfragerinnen und Nachfrager) mit einer Mitarbeiterin oder einem Mitarbeiter mit Kundenkontakt im Kontext eines Dienstleistungsunternehmens (Anbieter). Die zunehmende Digitalisierung hat dabei einen enormen Einfluss darauf, wie Dienstleistungen bereitgestellt werden. Die COVID-19-Pandemie in 2020 und 2021 hat diesen Trend verstärkt und zu einer Turbo-Digitalisierung geführt. Dienstleistungen werden zunehmend digitaler und automatisierter. Serviceroboter sind dabei eine wichtige und interessante Möglichkeit der Digitalisierung von Dienstleistungen.

Serviceroboter sind aktuell vor allem für Aufgaben mit einem hohen Volumen, einer geringen Heterogenität sowie einer geringen emotionalen Komplexität geeignet. Vom kognitiv-analytischen Niveau meistern Serviceroboter sowohl einfache als auch komplexe Aufgaben. Serviceroboter können dabei physisch oder virtuell sowie humanoid oder nicht-humanoid gestaltet sein. Es gibt aus der Perspektive der Kundinnen und Kunden, der Mitarbeiterinnen und Mitarbeitern sowie des Managements verschiedene Chancen, Herausforderungen und Handlungsempfehlungen. Insgesamt kann festgehalten werden, dass Serviceroboter zunehmend dort eingesetzt werden, wo sie Serviceleistungen deutlich günstiger und besser erbringen können. Technische Entwicklungen lassen dabei in Zukunft immer neue Einsatzfelder und Anwendungsmöglichkeiten entstehen, auf die sich vorbereitet werden sollte. 